\title{
ON THE EQUATIONS OF MOTION IN A RIEMANN SPACE
}

\section{S. KNEBELMAN}

Introduction. The object of this note is to give a geometric interpretation to the conditions of integrability of Killing's equations ${ }^{1}$ and to the similar equations for collineations ${ }^{2}$ in an affinely connected space. This is done by considering the more general problem of mapping a space upon itself, the mapping preserving some differential invariant. We shall be concerned with purely local properties so that the functions involved are assumed to have continuous derivatives of any necessary order and the groups are assumed to be infinitesimal.

1. Infinitesimal mapping of $V_{\mathfrak{n}}$ upon itself. By an infinitesimal mapping of $V_{n}$ upon itself we understand a correspondence generated by a vector field $\xi^{i}(x)$; that is, to a point $P(x)$ corresponds a point $P(\bar{x})$ where

$$
\bar{x}^{i}=x^{i}+\xi^{i}(x) \delta t .
$$

In order to see the effect of such a mapping on a differential invariant, we consider - merely to be specific - a tensor field of components $T^{i}{ }_{j}(x)$. Such a tensor field is mapped into one whose components are

$$
\bar{T}^{i}{ }_{j}(\bar{x})=\frac{\partial \bar{x}^{i}}{\partial x^{\alpha}} \cdot \frac{\partial x^{\beta}}{\partial \bar{x}^{j}} T_{\beta}^{\alpha}(x),
$$

which for the mapping (1.1) gives

$$
\bar{T}_{j}^{i}(\bar{x})=T^{i}{ }_{j}(x)+\left(\frac{\partial \xi^{i}}{\partial x^{\alpha}} T^{\alpha}{ }_{j}-\frac{\partial \xi^{\beta}}{\partial x^{j}} T^{i}{ }_{\beta}\right) \delta t .
$$

By the variation of $T^{i}{ }_{j}(x)$ we shall understand $\lim _{\delta t \rightarrow 0}\left(T^{i}{ }_{j}(\bar{x})\right.$ $\left.-T^{i}{ }_{j}(\bar{x})\right) / \delta t$ and shall denote it by $\delta T^{i}{ }_{j}(x) / \delta t$. Then (1.2) gives

$$
\frac{\delta T^{i}{ }_{j}(x)}{\delta t}=\xi^{\alpha} \frac{\partial T^{i}{ }_{j}}{\partial x^{\alpha}}-\frac{\partial \xi^{i}}{\partial x^{\alpha}} T^{\alpha}{ }_{j}+\frac{\partial \xi^{\alpha}}{\partial x^{j}} T_{\alpha}^{i},
$$

with a corresponding expression for any scalar or tensor field. If a tensor field is to remain invariant, it is necessary and sufficient that its variation be zero. From (1.3) it is obvious that a zero tensor field

Received by the editors May 29, 1945.

${ }^{1}$ L. P. Eisenhart, Riemannian geometry, chap. 6.

${ }^{2}$ L. P. Eisenhart, Non-Riemannian geometry, chap. 3 ; our $R^{i}{ }_{j k l}$ is the negative of that used by Eisenhart. 
is invariant under an arbitrary mapping; it is also evident that the variation is a differential invariant. Hence if the space $V_{n}$ is affinely connected, partial differentiation may be replaced by covariant differentiation.

2. Affinely connected $V_{n}$. Denoting covariant differentiation by $T_{, k}$ we have from (1.3)

and

$$
\begin{aligned}
\left(\frac{\delta T^{i}{ }_{j}}{\delta t}\right)_{, k}= & \xi^{\alpha} T^{i}{ }_{j, \alpha, k}+\xi^{\alpha}{ }_{, k} T^{i}{ }_{j, \alpha}+\xi^{\alpha}{ }_{, j, k} T^{i_{\alpha, k}} \\
& -\xi^{i}{ }_{, \alpha, k} T^{\alpha}{ }_{j}-\xi^{i}{ }_{, \alpha} T^{\alpha}{ }_{j, k}+\xi^{\alpha}{ }_{, j} T^{i}{ }_{\alpha, k}
\end{aligned}
$$

$$
\frac{\delta\left(T^{i}{ }_{j, k}\right)}{\delta t}=\xi^{\alpha} T^{i}{ }_{j, k, \alpha}+\xi^{\alpha}{ }_{, j} T^{i}{ }_{\alpha, k}+\xi^{\alpha}{ }_{, k} T^{i_{j, \alpha}}-\xi^{i}{ }_{, \alpha} T^{\alpha, k}{ }_{j, k} ;
$$

subtracting and using Ricci's identities we get

$$
\begin{aligned}
& \left(\frac{\delta T^{i}{ }_{j}}{\delta t}\right)_{, k}-\frac{\delta\left(T^{i}{ }_{j, k}\right)}{\delta t} \\
& =T^{i}{ }_{\alpha}\left(\xi^{\alpha},{ }_{j, k}+\xi^{\beta} R^{\alpha}{ }_{j k \beta}\right)-T^{\alpha}{ }_{j}\left(\xi^{i}, \alpha, k+\xi^{\beta} R^{i}{ }_{\alpha k \beta}\right),
\end{aligned}
$$

where $R$ is the curvature tensor of the $V_{n}$. We recall ${ }^{2}$ that

$$
\xi^{\alpha},{ }_{j, k}+\xi^{\beta} R^{\alpha}{ }_{j k \beta}=0
$$

are precisely the differential equations of an affine collineation. Hence we have the following theorem.

THEOREM 1. A necessary and sufficient condition that a mapping must satisfy so that variation and covariant differentiation be commutative is that the mapping be an affine collineation.

The conditions of integrability of (2.2) may be written ${ }^{2}$ as

$$
\begin{aligned}
& \xi^{\alpha} R^{i}{ }_{j k l, \alpha}-R^{\alpha}{ }_{j k l} \xi^{i}{ }_{, \alpha}+R^{i}{ }_{\alpha k l} \xi^{\alpha}{ }_{, j}+R^{i}{ }_{j \alpha l} \xi^{\alpha}{ }_{, k}+R^{i}{ }_{j k \alpha} \xi^{\alpha}{ }_{, l}=0, \\
& \xi^{\alpha} R^{i}{ }_{j k l, m, \alpha}-R^{\alpha}{ }_{j k l, m} \xi^{i}, \alpha+R_{\alpha k l, m}^{i} \xi^{\alpha}, j+\cdots=0,
\end{aligned}
$$

and from (1.3) it follows that they may be written as

$$
\delta R^{i}{ }_{j k l} / \delta t=0, \delta R^{i}{ }_{j k l, m} / \delta t=0, \cdots .
$$

Hence we have the following theorem.

THEOREM 2. A necessary and sufficient condition that an affinely connected space must satisfy to admit a group of collineations is that it admit 
a group of mappings leaving the curvature tensor and its successive covariant derivatives invariant.

From the theory of differential equations of the form (2.2) and from (2.3) it is evident that

THEOREM 3. The group of affine collineations is a subgroup of mappings preserving the curvature tensor and its successive covariant derivatives.

That the two groups are not necessarily identical can be seen from the case of an affinely flat space. Since $R^{i}{ }_{j k l}=0$ in this case the group of mappings is the group of analytic transformations while the group of collineations is (equivalent to) the group of linear transformations. On the other hand if $\mathcal{S H}_{r}$, the group of collineations, is of generic rank $r$, then, as can be shown, the group of mappings is also (S) $_{r}$.

3. Motion in a Riemann space. If in a metric space, with metric tensor $g_{i j}$, we require that the mapping preserve this tensor, we get according to (1.3)

$$
g_{i \alpha} \xi^{\alpha}, j+g_{\alpha j} \xi^{\alpha}, i=0,
$$

which are the equations of Killing for an infinitesimal motion. If they hold then (2.2) and (2.3) necessarily follow. ${ }^{1}$ We consider a space of constant (nonzero) curvature. In this case

$$
R^{i}{ }_{j k l}=c\left(\delta^{i}{ }_{k} g_{j l}-\delta^{i}{ }_{l} g_{j k}\right)
$$

and since $R^{i}{ }_{j k l, m}=0$ in this case, (2.3) reduce to

$$
\delta^{i}{ }_{k}\left(g_{\alpha l} \xi^{\alpha}{ }_{, j}+g_{j \alpha} \xi^{\alpha}, l\right)-\delta^{i}{ }_{l}\left(g_{\alpha k} \xi^{\alpha},{ }_{j}+g_{j \alpha} \xi^{\alpha}, k\right)=0
$$

which by contraction give (3.1). Hence we have the following theorem.

THEOREM 4. In a space of constant (nonzero) curvature a mapping preserving curvature is a motion.

Since the group of motions in any case is a subgroup of the group of collineations it follows as a corollary of Theorem 4 that in a space of constant nonzero curvature the group of motions and the group of affine collineations are the same. A little more than this can be easily proved; this corollary is true for an Einstein space $V_{n}(n>2)$ which is not flat.

4. Motions as a subgroup of collineations. Suppose that a Riemannian $V_{n}$ admits a group $\mathfrak{S}_{r}$ of affine collineations $\left(r \leqq n^{2}+n\right)$. This 
means that (2.3) admit $r$ linearly independent solutions $\xi_{(\sigma)}^{i}$, $\sigma=1,2, \cdots, r$. Let the variation of $g_{i j}$ corresponding to $\xi^{i}{ }_{(\sigma)}$ be denoted by $h^{(\sigma)}{ }_{i j}$ (cf. (3.1)). Then the question is whether there exist $r$ constants $c^{(\sigma)}$ such that $c^{(\sigma)} \xi^{i}{ }_{(\sigma)}$ is a motion; that is, such that

$$
c^{(\sigma)} h^{(\sigma)}{ }_{i j}=0 \text {. }
$$

Obviously a necessary condition is that the matrix of $n(n+1) / 2$ rows and $\mathrm{r}$ columns whose elements are $h^{(\sigma)}{ }_{i j}$ be of rank less than $r$. We shall show that this condition is also sufficient. For suppose this rank is $s<r$; then (4.1) admit $r-s$ linearly independent solutions $\phi_{\alpha}^{(\sigma)}(x), \alpha=1,2, \cdots, r-s$, so that

$$
\phi_{\alpha}^{(\sigma)} h_{i j}^{(\sigma)}=0
$$

are identities in $x$. Hence

$$
\phi_{\alpha, k}^{(\sigma)} h_{i j}^{(\sigma)}+\phi_{\alpha}^{(\sigma)} h_{i j, k}^{(\sigma)}=0
$$

and because of (2.2), $h^{(\sigma)}{ }_{i j, k}=0$. Hence $\phi^{(\sigma)}{ }_{\alpha, k}$ is also a solution of (4.1) and hence

$$
\phi_{\alpha, k}^{(\sigma)}=A_{\alpha k}^{\beta} \phi_{\beta}^{(\sigma)} .
$$

If there is to be a solution $c^{(\sigma)}$ then a set of $r-s$ functions $p^{\alpha}$ must exist such that

$$
c^{(\sigma)}=p^{\alpha} \phi_{\alpha}^{(\sigma)}
$$

from which it follows that

$$
\rho^{\beta}{ }_{, k} \phi^{(\sigma)}{ }_{\beta}+p^{\alpha} A_{\alpha k}^{\beta} \phi_{\beta}^{(\sigma)}=0 .
$$

Hence, since $\phi_{\alpha}^{(\sigma)}$ are linearly independent we must have

$$
p^{\beta}, k+p^{\alpha} A^{\beta}{ }_{\alpha k}=0 \text {. }
$$

From (4.3) and the linear independence of $\phi_{\beta}^{(\sigma)}$ it follows that

$$
A_{\alpha k, l}^{\gamma_{\alpha k}}-A_{\alpha l, k}^{\gamma_{2}}+A_{\alpha k}^{\beta} A^{\gamma_{\beta l}}-A_{\alpha l}^{\beta} A^{\gamma_{\beta k}}=0
$$

and these are precisely the conditions of complete integrability of the differential equations (4.4). Hence (4.4) admit a set of $r-s$ linearly independent solutions; thus we have the following theorem.

THEOREM 5. If a Riemann space admits a group $\mathbf{S}_{r}$ of affine collineations a necessary and sufficient condition that it admit a group of motions is that the matrix $\left\|h^{(\sigma)}{ }_{i j}\right\|$ be of rank less than $r$. If this rank is $s$, then the group of notions is of order $r-s$.

State College of Washington 